\title{
Examining the Free Radical Bonding Mechanism of Benzoquinone- and Hydroquinone-Methanol Passivation of Silicon Surfaces
}

\author{
Nicole A. Kotulak ${ }^{\mathrm{a}, 1, *}$, Meixi Chen ${ }^{\mathrm{b}}$, Nikolas Schreiber, Kevin Jones ${ }^{\mathrm{c}}$, \\ Robert Opilac \\ University of Delaware, Newark, Delaware 19716 \\ U.S.A. \\ ${ }^{a}$ Department of Electrical and Computer Engineering \\ ${ }^{b}$ Department of Chemistry \\ ${ }^{c}$ Department of Materials Science and Engineering
}

\begin{abstract}
The surface passivation of p-benzoquinone (BQ) and hydroquinone (HQ) when dissolved in methanol (ME) have been examined through effective lifetime testing of crystalline silicon (c-Si) wafers treated with the aforementioned solutions. Changes in the availability of both photons and protons in the solutions were demonstrated to affect the level of passivation achieved. The requirement of both excess protons and ambient light exposure to maintain high effective lifetimes supports the presence of a free radical species that drives the surface passivation. Surface analysis suggests a 1:1 ratio of HQ-like bonds to methoxy bonds on the c-Si surface after treatment with a $\mathrm{BQ} / \mathrm{ME}$ solution.
\end{abstract}

Keywords: benzoquinone, methanol, quinhydrone, hydroquinone, surface passivation, silicon, effective lifetime, free radical, $\mathrm{QH}^{\bullet}$

\footnotetext{
*Corresponding author.

Email address: nicole.kotulak.ctr@nrl.navy.mil (Nicole A. Kotulak)

${ }^{1}$ Present address: Naval Research Laboratory, Washington, D.C. 20357, United States
} 


\section{Introduction}

Crystalline silicon (c-Si) is a dominant material in photovoltaic (PV) devices, and the treatment of wafer surfaces to reduce detrimental surface recombination effects is critical to c-Si-based device performance. To date, the predominant methods for surface passivation require processes that elevate the system temperature for material deposition. Room temperature solutionbased methods, however, provides a rapid, less-intensive means to electrically passivate c-Si surfaces. The molecular mixture quinhydrone (QHY), which is a 1:1 mixture of p-benzoquinone (BQ) and hydroquinone (HQ), as shown in Fig. 1, has been demonstrated to passivate c-Si surfaces, and solutions of QHY constituents in methanol (ME) that have been examined in order to more fully understand the bonding mechanisms involved [1]. If the mechanisms underlying this high-lifetime passivation method can be determined, the information can be used to engineer materials that will provide the same functionality for the c-Si surface, but with other, device-specific functions. The capability to combine c-Si surface passivation with specified electrical properties, such as electron or hole blocking, high carrier mobility, or transparency to a specific wavelength range has a direct bearing on the growing field of hybrid organic-inorganic PV devices, or any device system considering the implementation of alternative engineered materials.

While much work has been performed to demonstrate the efficacy of QHY/ME solutions, the mechanisms behind the high-quality passivation and the degradation of the effect once the passivated c-Si sample is removed from solution are still unknown $[2,3,4,5,6,7,8,9]$. Work on separate solutions of the constituent species of QHY have shown BQ/ME outperforms HQ/ME, but that HQ/ME passivated surfaces will improve given more than 3 hours of exposure to solution $[6,10]$. These studies use effective lifetime to monitor and evaluate the performance of the passivants.

Surface analysis has also been performed using fourier transform infrared spectroscopy (FTIR). These studies indicate that surfaces treated with QHY/ME, $\mathrm{BQ} / \mathrm{ME}$, and HQ/ME all display Si-methoxy and Si-HQ bond vibrations, with little if any noticeable $\mathrm{Si}-\mathrm{O}$ bonds present [10]. Due to the Si surface termination with $\mathrm{HQ}$-like molecules, it has been suggested that BQ converts into the free radical $\mathrm{QH}^{\bullet}$ through the abstraction of a $\mathrm{H}$, and it is this free radical that bonds to a Si dangling bond [10, 11]. For HQ solutions, it is proposed that HQ first converts to BQ, an already demonstrated process $[10,12,13,14]$. 


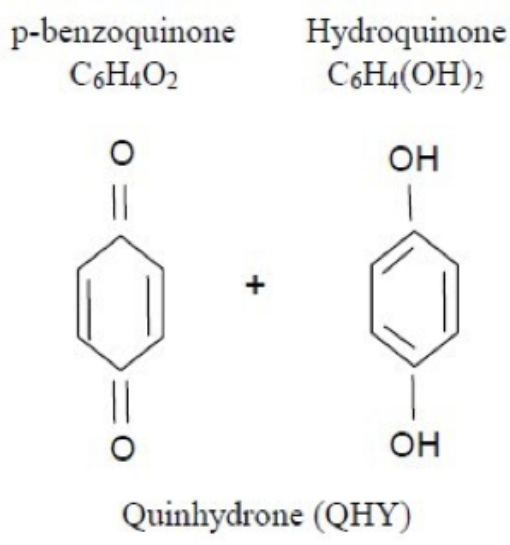

Figure 1: Constituent molecules of the Quinhydrone compound.

In this work, the existence of the proposed free radical $\mathrm{QH}^{\bullet}$ in this particular passivation system will be examined through effective lifetime measurements of solution passivated c-Si. The importance of protons and photons to the passivation mechanism - necessary components in the conversion process of $\mathrm{QH}^{\bullet}$ - will be demonstrated. The surface chemistry of c-Si after passivation with $\mathrm{BQ} / \mathrm{ME}$ will also be examined in order to support the $\mathrm{QH}^{\bullet}$ hypothesis, and to determine the ratio of organic molecules present.

\section{Experimental}

The metric used for determining the effectiveness of surface passivation is effective lifetime $\left(\tau_{\text {eff }}\right)$, which is measured by contactless photoconductance decay (PCD) using a WCT-120 from Sinton Consulting, Inc., with upgraded hardware to measure high resistivity wafers $[15,16]$. General mode was used, which combines quasi-steady-state and transient measurement analysis for more flexible data collection. Due to the contactless nature of the measurement, $\tau_{\text {eff }}$ could be measured while the Si wafer was still in the passivating solution. An optical constant of 0.7 corresponding to wafer thicknesses between 100 and $400 \mu \mathrm{m}$ thick with no coating on the surface was used for the generalized analysis [17].

Two wafer types were tested with the following specifications: 1) n-type, P-doped, $<100>$, double-side polished (DSP), $500 \mu \mathrm{m}$ thick, 20-30 $\Omega$-cm, 2" x 2", and 2) p-type, B-doped, <100>, DSP, $525 \mu \mathrm{m}, 10 \mathrm{k} \Omega$-cm, and 2" x 2". 
Wafer preparation included a surface clean, hydrogen termination, and organic surface passivation. The wafers were cleaned using a three-step process: 5 minutes in 4:1 Piranha at approximately $100^{\circ} \mathrm{C}, 5$ minutes in $\mathrm{DI} \mathrm{H}_{2} \mathrm{O}$, and 2 minutes in dilute $\mathrm{HF}$ at 1:50 $\mathrm{HF}: \mathrm{DI} \mathrm{H}_{2} \mathrm{O}$.

After cleaning, the wafers were immediately immersed into the appropriate passivation solution that had been mixed in a clear, acid-proof bag with a zip closure. The use of a bag, rather than a dish, allows for accurate lifetime measurements without the need for system recalibration. The solutions were prepared with a methanol (ME) (J.T. Baker CMOS grade, as-is) solvent, unless otherwise noted. The organic molecules p-Benzoquinone (BQ) (98+\% Alfa Aesar, as-is) and Hydroquinone (HQ) (99\% Acros Organics, asis) were used as the passivants, and were mixed with the ME to produce $0.1 \mathrm{M}$ solutions of $\mathrm{BQ} / \mathrm{ME}$ and $\mathrm{HQ} / \mathrm{ME}$, respectively.

Lifetime measurements began with $t=0$ indicating the first measurement taken immediately after wafer immersion into the passivation solution. The effective lifetime was measured and recorded every 15 minutes, unless otherwise noted, for a period of 2 to 24 hours, depending upon the sample, and the wafer remained immersed in solution for the duration of the experiment. The measured photoconductance is only that of the wafer, as the solution has a negligible photoconductance. The length of time over which effective lifetime was measured will be noted where applicable. Unless otherwise noted, $\tau_{\text {eff }}$ is reported at a minority carrier density of $1 e 15 \mathrm{~cm}^{-3}$.

Varied lighting conditions were implemented in order to observe the effects of light on the passivation reaction. A light-proof enclosure was built around the tester, providing the ability to eliminate ambient light from some experiments and expose the wafer to light only during the brief flash for carrier excitation. The following lighting conditions were examined:

1) Full Light - The entire measurement preparation process was performed in full ambient laboratory light conditions.

2) Partial Light - The passivation solution was prepared in full ambient laboratory light conditions. After cleaning and H-termination of the surfaces, the c-Si wafer was immersed into the passivation solution in an environment absent of light. The PCD measurements were taken in a light-proof enclosure, and the sample remained in said enclosure for the full duration of the experiment (2-24 hours). The solution and immersed wafer were exposed to light only during the illuminating flash for each measurement.

3) No Light - The passivation solution was mixed in the absence of light, followed by wafer immersion, also in the absence of light. The PCD measure- 
ments were taken in a light-proof enclosure, and the sample remained in said enclosure for the full duration of the experiment (2-24 hours). Again, the solution and immersed wafer were exposed to light only during the illuminating flash for each measurement.

In order to evaluate the relative magnitude of the light dose from the PCD flash when compared with ambient light, a Sekonic L-358 light meter was used to determine the lux of the light sources. To calculate the final dose from each source, the lux was multiplied by the duration of the exposure over a one hour timeframe. The result is that the PCD flash accounts for $2 \%$ of the total light dose when compared with the ambient light dose over a one hour period.

A PHI model 5600 laboratory X-ray photoelectron spectrometer (XPS) equipped with AlK $\alpha$ mono source was used to survey the deeper core states. Analysis was taken at a base pressure of $8 \times 10^{-10}$ Torr, where each region was taken at a pass energy of $23.50 \mathrm{eV}$ with $0.2 \mathrm{eV} /$ step at a spot size of $800 \mu \mathrm{m}$ at a collection angle of $45^{\circ}$. All fitting was done with a PHI Matlab code version V3.0. This program used the general forms of the GaussianLorentzian sum (GLS) for the peak fitting.

\section{Results \& Discussion}

\subsection{Active Bonding Constituent and Time Effects in $Q H Y / M E$ Solutions}

For QHY/ME passivation, literature suggests that BQ is the active passivant, and that the passivation effects of HQ improve over prolonged exposure of the wafer to the solution $[6,10]$. This is confirmed under Full Light conditions on both n- and p-type wafers, using a clean Si wafer immersed in ME as a control sample.

As can be seen in Fig. 2, the BQ/ME solution provides an immediate, high lifetime passivation. The HQ/ME solution does not have the same immediate effect as BQ/ME, performing only slightly better than the control solution within the first 2 hours of wafer immersion. At 24 and 48 hours, however, the lifetimes of n-type samples passivated with HQ/ME greatly improve, indicating an increased passivation capability over time.

In order to further evaluate the change in passivation capability of $\mathrm{HQ} / \mathrm{ME}$ over time, p- and n-type wafers were immersed in HQ/ME, and lifetimes were measured every 15 minutes under Full Light conditions for a duration of 14 hours (n-type) to 20 hours (p-type) - the period of time required for the life-

time to reach asymptotic behavior. The changes in lifetime as a function of 


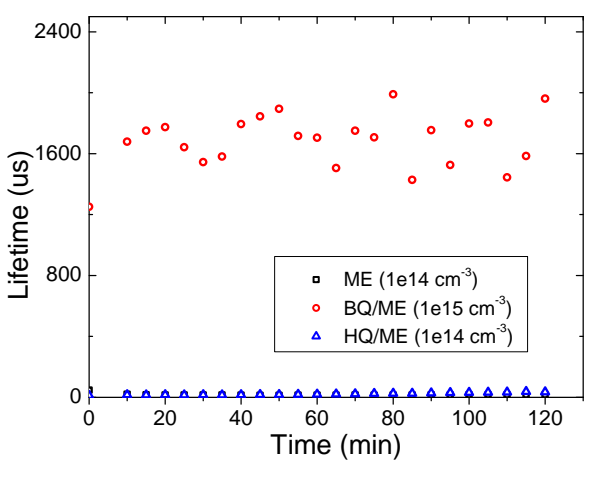

(a)

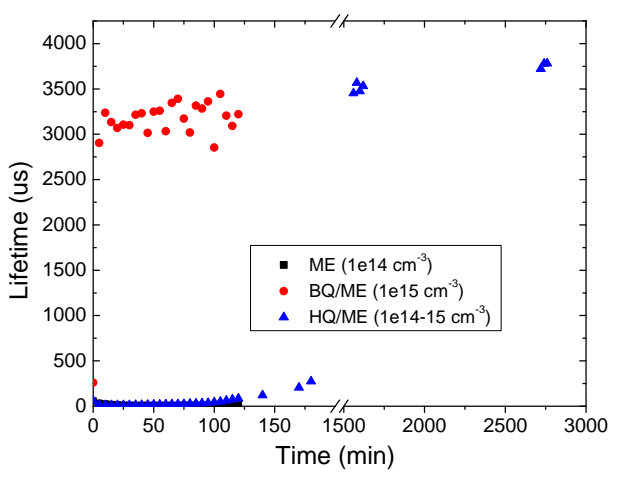

(c)

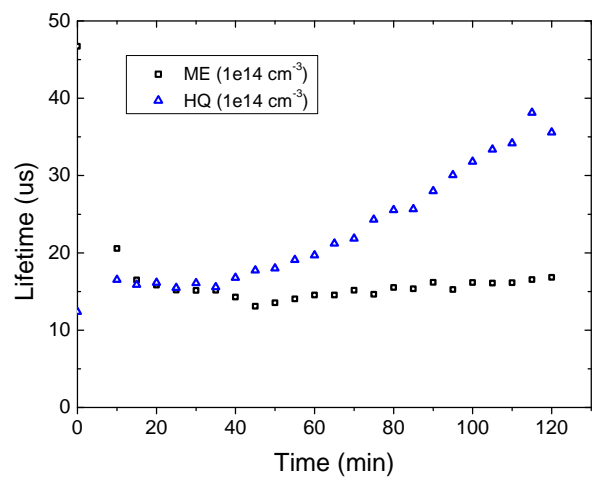

(b)

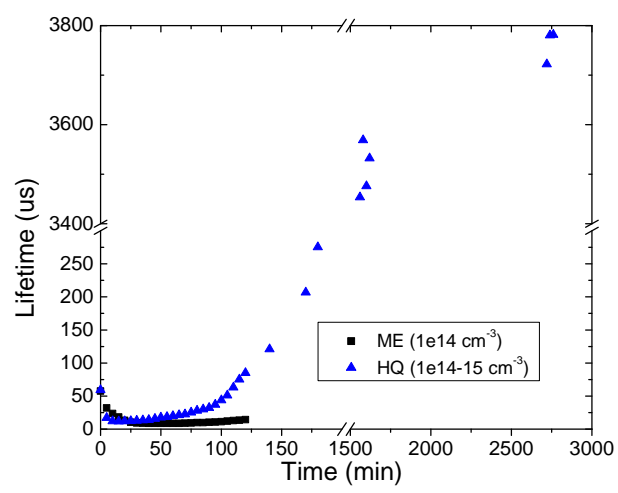

(d)

Figure 2: The change in lifetime a) over 2 hours for a p-type wafer in BQ/ME, HQ/ME, and a control solution, $\mathrm{ME}, b$ ) over 2 hours for a p-type wafer in $\mathrm{HQ} / \mathrm{ME}$ and $\mathrm{ME}, c$ ) over 2 hours for an n-type wafer in $\mathrm{BQ} / \mathrm{ME}, \mathrm{HQ} / \mathrm{ME}$, and ME, where the HQ/ME sample also has data points at 24 and 48 hours, and $d$ ) over 48 hours for an n-type wafer in HQ/ME and ME. The minority carrier density at which lifetime is reported is noted in the legends.

time for wafer immersion are shown in Figs. 3(a) and 3(b) for n- and p-type wafers, respectively. 


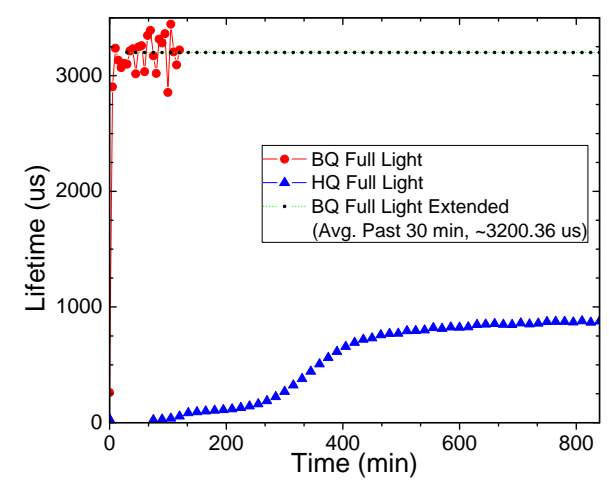

(a)

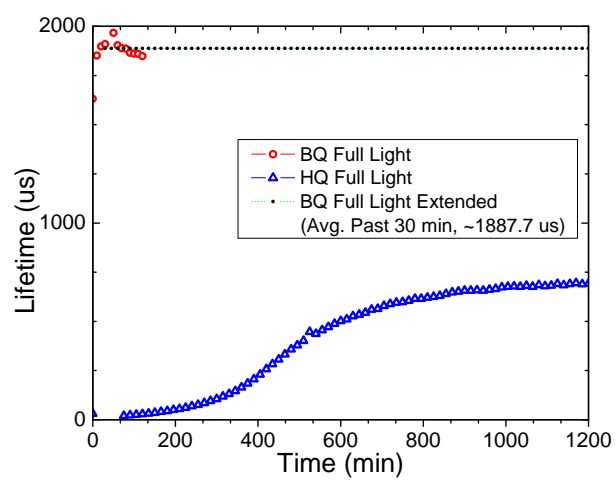

(b)

Figure 3: Change in lifetime during exposure of a) an n-type wafer to HQ/ME for 14 hours, and b) a p-type wafer to HQ/ME for 20 hours.

The resulting change in lifetime for samples passivated in HQ/ME follows a similar trend to that seen in the literature, with the initial 2 hours of passivation showing negligible improvements, and rapid increases in lifetimes at 70-120 minutes of exposure to solution [10]. The lifetimes of p-type substrates improve at a slower rate than those of n-type substrates, which explains the lack of observed improvement after 2 hours for earlier experimentation on p-type substrates.

Confirmation of the lifetime improvement for HQ/ME passivated substrates suggests a time-dependent reaction within the $\mathrm{HQ} / \mathrm{ME}$ solution that converts the HQ into a more effective passivating species, originally believed to be BQ. The mechanism through which this proposed conversion occurs, and a reevaluation of the active passivating species, is the next component of the study.

\subsection{Free Radical Bonding Species $Q H^{\bullet}$}

BQ and HQ will convert back and forth in solution through an intermediary third species $-\mathrm{QH}^{\bullet}$ - which has been demonstrated in the literature, and is shown in Fig. 4 [12, 13, 14]. This conversion pathway not only provides a 
mechanism by which HQ can convert to the more active BQ molecule, it also allows for the presence of a third molecular species in solution that, while having a short life in solution, may also prove to be an active passivating species. This free radical would be consistent with both our findings and those in literature. It has been demonstrated that BQ is the active passivating species relative to $\mathrm{HQ}$, however, BQ lacks the $\mathrm{OH}$ bond of the HQ-like structures observed in surface analysis after passivation [10]. It is necessary, therefore, for BQ to convert into a species that does have an $\mathrm{OH}$ bond in order for the lifetime results to correlate with the surface analysis. In addition, HQ does have the necessary $\mathrm{OH}$ bonds, but lacks the high lifetime of a good passivation, which indicates that $\mathrm{HQ}$ must also undergo a conversion to a more active configuration. $\mathrm{QH}^{\bullet}$ has the requisite configuration, and the focus will now turn to demonstrating that $\mathrm{QH}^{\bullet}$ is both present in the system and the most likely passivating species.

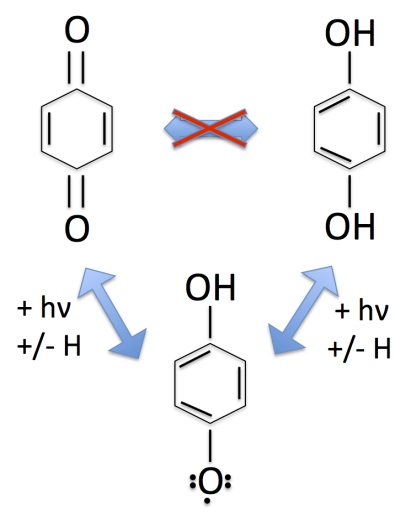

Figure 4: Suggested conversion pathway between BQ and HQ through the intermediary free radical $\mathrm{QH}^{\bullet}$.

As can be seen in Fig. 4, the conversion to/from $\mathrm{QH}^{\bullet}$ requires photons and either the addition or subtraction of a proton. In order to support the existence of $\mathrm{QH}^{\bullet}$ and its role in the effectiveness of the passivation technique, changes in lifetime will be examined as a function of both available protons and photons.

\subsubsection{Importance of Protons to the Passivation Mechanism}

Determining the effect of protons on the passivation mechanism required a solvent change from $\mathrm{ME}\left(\mathrm{CH}_{3} \mathrm{OH}\right)$, which is known to have a labile proton on 
the alcohol oxygen. If the free radical $\mathrm{QH}^{\bullet}$ were indeed an active component of the system, the extra protons from ME would facilitate the conversion. Removal of the excess protons, therefore, should display a decrease in the passivation capability of the solution if $\mathrm{ME}$ were to be replaced by the aprotic solvent diethyl ether $\left(\mathrm{CH}_{3} \mathrm{CH}_{2} \mathrm{OCH}_{2} \mathrm{CH}_{3}\right)$. Additionally, the removal of the labile proton from $\mathrm{ME}$ would leave a methoxy free radical, which can also bond to the c-Si surface. This is likely the source of the methoxy bonds observed after surface analysis, which will be discussed in Section 3.2.3. To compare the changes in lifetimes for ME versus diethyl ether solvents, wafers immersed in BQ/ME were compared with those immersed in BQ/diethyl ether. The resulting changes in lifetimes over a period of two hours are shown in Fig. 5, and display the anticipated decrease in performance for those wafers passivated in the solution using an aprotic solvent. It is also noted that the lifetimes in ether were improving overtime, indicating the possibility of $\mathrm{H}_{2} \mathrm{O}$ contamination of the solvent over the duration of the experiment, with $\mathrm{H}_{2} \mathrm{O}$ acting as a source of additional protons. To test this possibility, solutions using anhydrous ME were compared with those of anhydrous ME contaminated with $2 \%$ by volume $\mathrm{H}_{2} \mathrm{O}$, and the resulting lifetime data showed no significant difference in passivation performance. With the elimination of $\mathrm{H}_{2} \mathrm{O}$ contamination as the source of excess protons, it should be noted that another source exists - the H-terminated Si surface. While this mechanism is not examined here, there is a high probability that the removal of $\mathrm{H}$ atoms from the Si surface provides the necessary protons in the aprotic solutions.

\subsubsection{Effect of Light on the Passivation Mechanism}

The formation of $\mathrm{QH}^{\bullet}$ requires protons and photons. The former has been shown to affect the system by solvent substitution. The latter will be evaluated using a change in lighting conditions as described in Section 2. Passivation by $\mathrm{BQ} / \mathrm{ME}$ is an almost immediate reaction, while passivation by $\mathrm{HQ} / \mathrm{ME}$ requires a much longer period of time. If the conversion between either of these constituent molecules and $\mathrm{QH}^{\bullet}$ is occurring, the $\mathrm{BQ} / \mathrm{ME}$ conversion to $\mathrm{QH}^{\bullet}$ is a much faster reaction. If ambient light is reduced or removed from the system during the passivation reaction, the differing time scale for conversion should become more apparent. As light can never be completely eliminated due to the nature of the measurement technique, however, some $\mathrm{QH}^{\bullet}$ conversion is to be expected. The change in lifetime resulting from altered ambient light exposure is presented in Fig. 6.

For all cases, samples passivated under Full Light conditions had higher 


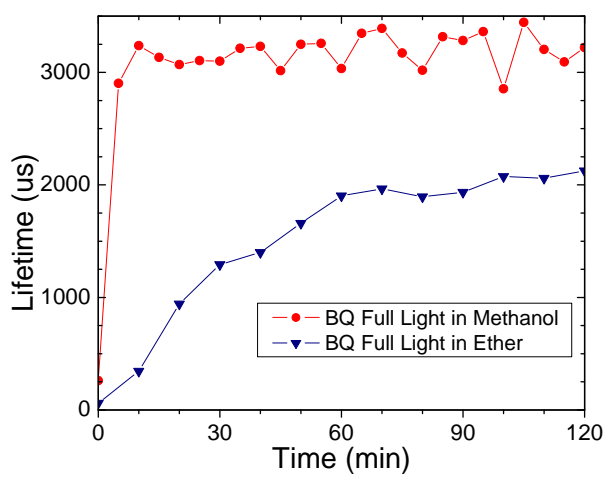

(a)

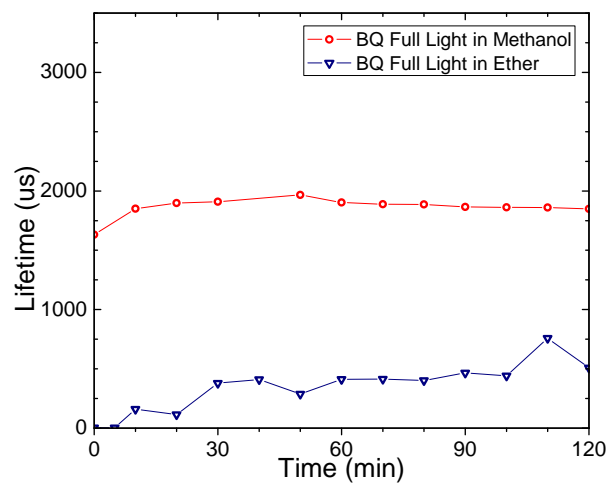

(b)

Figure 5: Comparison of lifetimes of a) n-type wafers and b) p-type wafers in BQ/ME versus $\mathrm{BQ} /$ Ether.

lifetimes, which indicates that the bonding mechanism for high quality passivation requires continual exposure to light when the sample is in contact with the solution. The BQ/ME solution shows comparable lifetimes between the Partial and No Light samples, both of which have significantly lower lifetimes than the Full Light samples. For HQ/ME solutions, however, Partial Light produces a higher lifetime than No Light, both of which underperform solutions under Full Light conditions.

Referring to Fig. 4, there are two pathways for conversion to/from $\mathrm{QH}^{\bullet}$. Based on the immediate effects of $\mathrm{BQ} / \mathrm{ME}$, the conversion from $\mathrm{BQ}$ to $\mathrm{QH}^{\bullet}$ is a fast conversion, on the order of nanoseconds [14]. Presumably, the free radical will convert back into $\mathrm{BQ}$ within the same timescale in the absence of a bonding site. Conversely, the conversion from $\mathrm{HQ}$ to $\mathrm{QH}^{\bullet}$ is a less likely reaction, based upon the slow rate of lifetime improvement from $\mathrm{HQ} / \mathrm{ME}$ solutions. Once $\mathrm{QH}^{\bullet}$ is present in a $\mathrm{HQ} / \mathrm{ME}$ solution, it will most likely revert along the more likely conversion pathway, becoming BQ rather than HQ.

This conversion mechanism explains the passivation behavior observed 


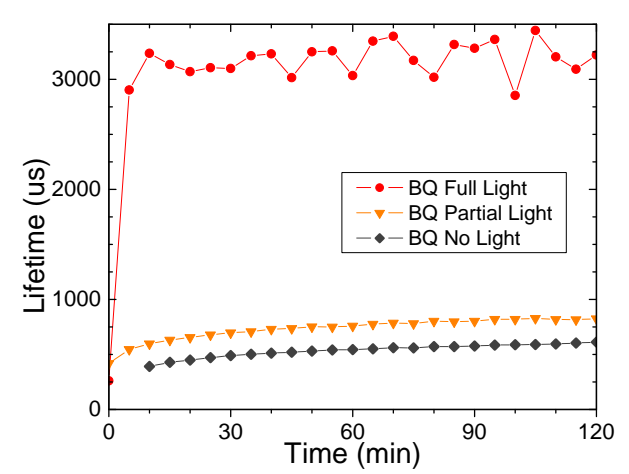

(a)

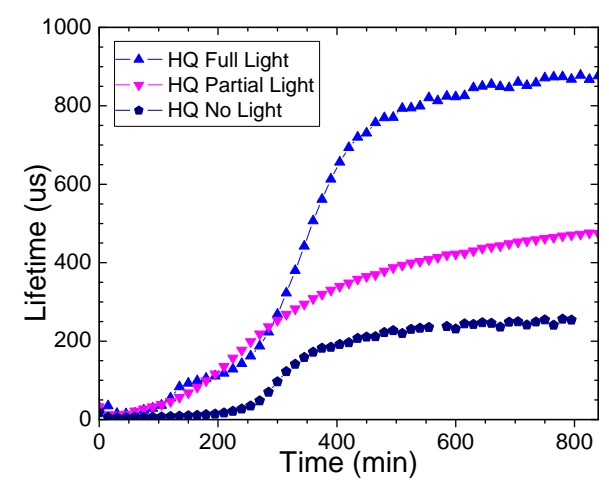

(c)

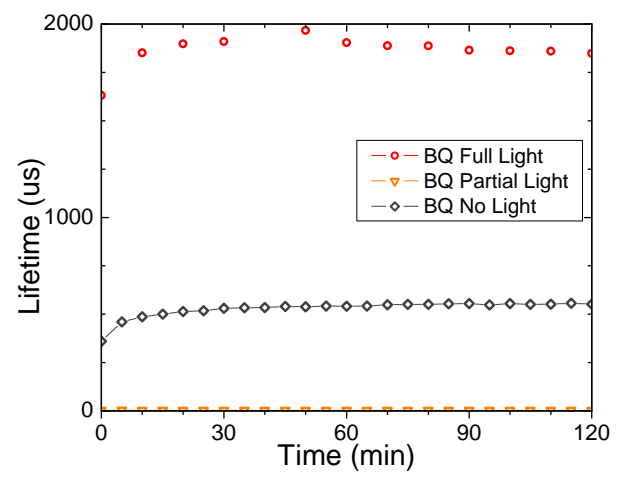

(b)

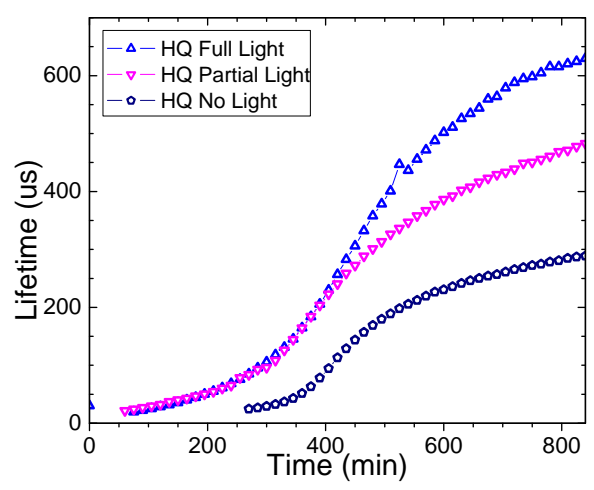

(d)

Figure 6: Comparison of different lighting conditions on the lifetimes of a) n-type wafers and b) p-type wafers in BQ/ME, and c) n-type wafers and d) p-type wafers in HQ/ME.

under different lighting conditions. Full Light conditions continually provide for the conversion of $\mathrm{BQ}$ and $\mathrm{HQ}$ into $\mathrm{QH}^{\bullet}$, with $\mathrm{HQ}$ taking longer to convert and build up enough $\mathrm{QH}^{\bullet}$ to bond with the surface and produce a noticeable difference in lifetime. The ambient light exposure has a significant impact, with the light exposure from the measurement flash accounting for only $2 \%$ 
of the total light dose over a one-hour period.

In $\mathrm{BQ} / \mathrm{ME}$, the solution under Partial Light initially forms $\mathrm{QH}^{\bullet}$ while under ambient light, but the free radical quickly reverts back into BQ by the time the wafer is in contact with the solution. Just like the No Light condition, then, $\mathrm{QH}^{\bullet}$ is only present after a flash from the test setup, and only the free radicals close enough to the Si surface form a bond. The remaining $\mathrm{QH}^{\bullet}$ reverts back into BQ. In HQ/ME, the Partial Light condition forms $\mathrm{QH}^{\bullet}$, which reverts into BQ rather than $\mathrm{HQ}$ when ambient light is no longer present. When the wafer is brought into contact with solution, then, there is a presence of both fast-acting $\mathrm{BQ}$ and the slower HQ species. For the No Light condition, however, the buildup of $\mathrm{BQ}$ from $\mathrm{QH}^{\bullet}$ has not occurred. Once there is light exposure in the system for each measurement, the conversion to $\mathrm{QH}^{\bullet}$ is faster for the Partial Light solution, resulting in higher lifetimes than the No Light solution. The outlier for this set of data is the Partial Light condition for a p-type wafer in $\mathrm{BQ} / \mathrm{ME}$, which repeatedly provides little to no passivation effect. There is no indication thus far of a reason for this discrepancy in performance.

\subsubsection{XPS Surface Analysis}

The change in lifetime under varying solution and light conditions suggests the molecular species that are bonding to the c-Si surface. In order to support this hypothesis, surface analysis was performed using XPS. The XPS spectra were obtained from c-Si wafers that had been passivated with $\mathrm{BQ} / \mathrm{ME}$ and left in solution under full ambient lighting conditions for 36 hours. The resulting spectra are shown in Figs. 7(a) $-7(\mathrm{c})$, and a representation of Si-methoxy and $\mathrm{Si}-\mathrm{QH}{ }^{\bullet}$ bonds is shown in Fig. 7(d).

Using the ratio of the bonds observed in Figs. $7(\mathrm{a})-7(\mathrm{c})$, the ratio of molecules bound to the c-Si surface can be identified as approximately 1:1 $\mathrm{QH}^{\bullet}$ to methoxy groups, where methoxy free radicals have bonded to the surface in addition to $\mathrm{QH}^{\bullet}$. In Fig. 7(a), the $\mathrm{O}$ 1s spectrum shows approximately 2:1 SiOC:OH. To address the ease with which Si develops a native oxide, Fig. 7(b) shows a very small $\mathrm{SiO}_{x}$ peak, indicating that there is little bonding between only $\mathrm{Si}$ and $\mathrm{O}$ at the c-Si surface. Finally, in Fig. 7(c), the ratio of $\mathrm{CO}$ to $\mathrm{CH}$ is approximately 3:4. Using Fig. 7(d) as a guide, all of these bond ratios correspond to the aforementioned surface condition of 1:1 $\mathrm{QH}^{\bullet}$ to methoxy. 


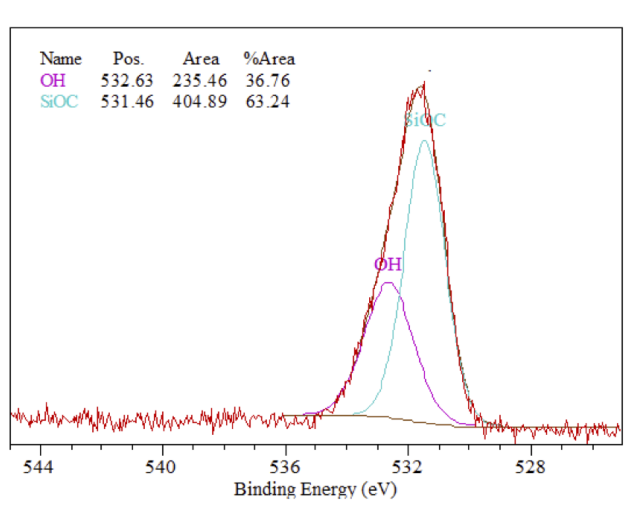

(a)

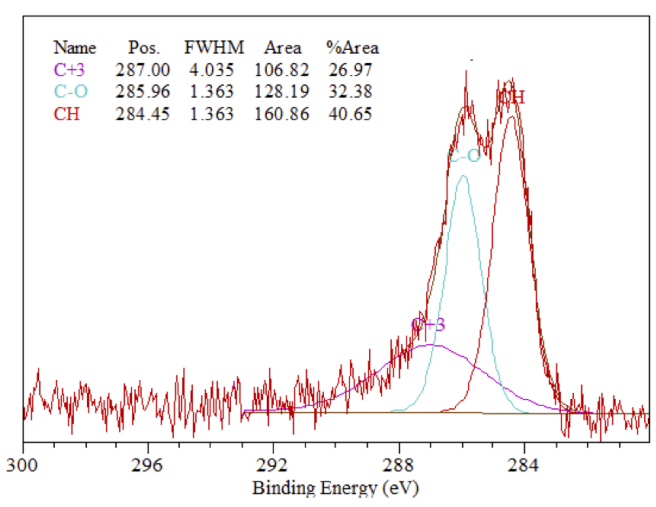

(c)

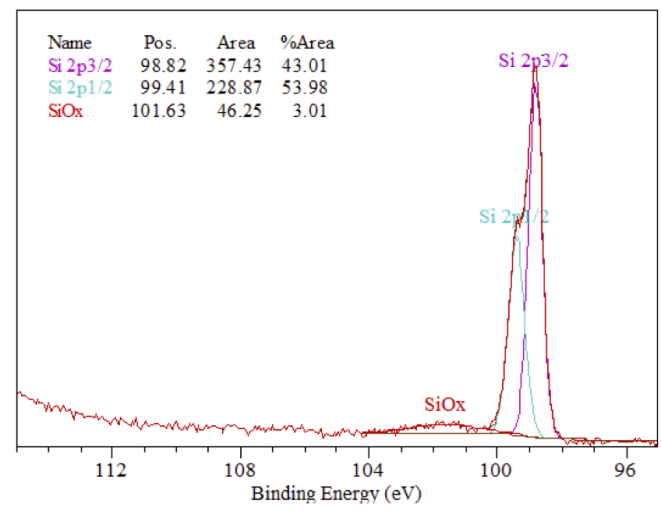

(b)

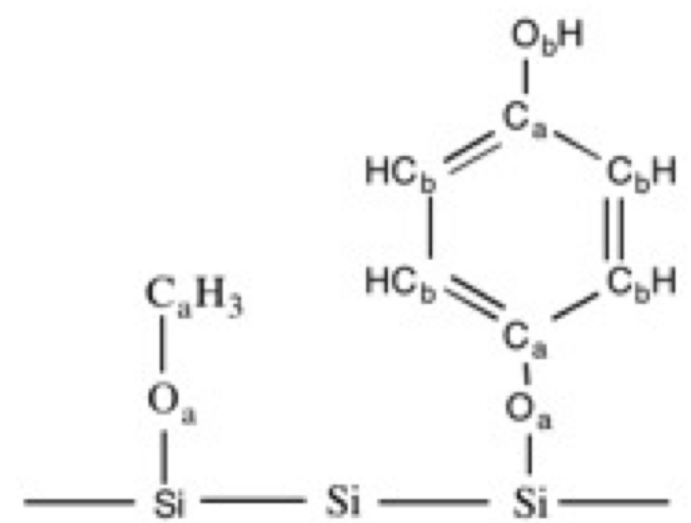

(d) Left: Si-methoxy, Right: Si-QH•

Figure 7: a) O 1s spectrum the $\mathrm{SiOC}$ and $\mathrm{OH}$ bond peaks, b) Si 2p spectrum showing the $\mathrm{Si}$ and $\mathrm{SiOx}$ bond peaks, and c) $\mathrm{C}$ 1s spectrum showing the $\mathrm{CH}, \mathrm{CO}$, and $-\mathrm{COOH}$ bond peaks for $\mathrm{BQ} / \mathrm{ME}$ on $\mathrm{H}$-terminated c-Si. d) A representation of the molecules bonded to the Si surface after passivation.

\section{Conclusion}

The passivation effects of QHY/ME constituent solutions on c-Si surfaces have been examined through a series of effective lifetime measurements and XPS surface analysis. It has been demonstrated that BQ is the active compo- 
nent of the QHY/ME solution, however, the efficacy of HQ/ME will improve after a long exposure time of the wafer to the solution. The free radical $\mathrm{QH}^{\bullet}$ is the likely passivating species, demonstrated through a systematic study of the effects of protons and light exposure on the passivation reaction. Finally, surface analysis through XPS has shown that there are both methoxy groups and $\mathrm{QH}^{\bullet}$ bound to the c-Si surface in an approximate 1:1 ratio.

\section{Acknowledgments}

This material is based upon work primarily supported by the National Science Foundation (NSF) and the Department of Energy (DOE) under NSF CA No. EEC-1041895. Any opinions, findings and conclusions or recommendations expressed in this material are those of the author(s) and do not necessarily reflect those of NSF or DOE.

[1] H. Matsuda, K. Osaki, I. Nitta, Bull. Chem. Soc. Jpn. 31 (1958) 611620 .

[2] H. Takato, I. Sakata, R. Shimokawa, Jpn. J. Appl. Phys. 40 (2001).

[3] H. Takato, I. Sakata, R. Shimokawa, Jpn. J. Appl. Phys. 41 (2002).

[4] B. Chhabra, S. Bowden, R. L. Opila, C. B. Honsberg, Appl. Phys. Lett. 96 (2010) 063502.

[5] B. Chhabra, S. Suzer, R. L. Opila, C. B. Honsberg, $33^{\text {rd }}$ IEEE Photovolt. Spec. Conf. (2008).

[6] R. L. Opila, D. Yang, N. Kotulak, L. Costello, B. Chhabra, 38th IEEE Photovoltaic Specialists Conference (2012) 2583-2587.

[7] M. R. Page, Q. Wang, T. H. Wang, Y. Yan, S. W. Johnston, T. F. Ciszek, $13^{\text {th }}$ Workshop on Crys. Si Solar Cell Mats. and Proc. (2003).

[8] H. Takato, I. Sakata, R. Shimokawa, $3^{\text {rd }}$ World Conf. on Photovolt. Energy Conv. (2003) 1108-1111.

[9] H. Takato, I. Sakata, R. Shimokawa, World Conf. on Photovolt. Energy Conv. (2006) 249-252. 
[10] R. Har-Lavan, R. Schreiber, O. Yaffe, D. Cahen, J. Appl. Phys. 113 (2013) 084909.

[11] T. F. Harper, M. J. Sailor, J. Am. Chem. Soc. 119 (1997) 6943-6944.

[12] S. M. Beck, L. E. Brus, J. Am. Chem. Soc. 104 (1982) 4789-4792.

[13] N. Akai, A. Kawai, K. Shibuya, J. Photochem. Photobiol. A 223 (2011) 182-188.

[14] R. Rossetti, L. E. Brus, J. Am. Chem. Soc. 108 (1986) 4718-4720.

[15] R. A. Sinton, A. Cuevas, Appl. Phys. Lett. 69 (1996) 2510-2512.

[16] R. A. Sinton, A. Cuevas, M. Stuckings, $25^{\text {th }}$ IEEE Photovolt. Spec. Conf. (1996) 457-460.

[17] Sinton Instruments, WCT-120 and Optional Suns-Voc User Manual (2012). 